\title{
Reducing drag on a flat plate subjected to incompressible laminar flow
}

\author{
A. AGRISS ${ }^{1, *}$, M. AGOUZOUL ${ }^{1,{ }^{* *}}$, A. ETTAOUIL ${ }^{1, * *}$ \\ ${ }^{1}$ Mohammed V University-Rabat, Mohammadia School of Engineers, Avenue Ibn Sina, P.O. Box 765-Agdal, \\ Rabat, Morocco \\ Mechanical and Energetic Engineering Research Team: Modeling and Experimentation ERG2(ME) \\ email addresses: *amineagriss@gmail.com, ${ }^{\star *}$ m.agouzoul@gmail.com, ${ }^{\star * *}$ ettaouil.ab@gmail.com
}

\begin{abstract}
:
The idea behind this work comes from the question: What is the impact of plate corrugations on drag? In this context, a numerical study of laminar incompressible flow over a flat plate and over corrugated plates is carried out. Numerical analysis is performed for low Reynolds numbers $(R e=10, R e=50, R e=100, R e=500, R e=1000)$ using the computational fluid dynamics (CFD) software ANSYS FLUENT. Simulations results are compared to each others and with those of the reference plate (flat plate (figure 4a)). Comparisons are made via drag coefficient $C_{d}$. This work is the beginning of a study that evaluates the impact of corrugations on drag reduction on a flat plate.
\end{abstract}

Keywords : drag, ANSYS FLUENT, flat plate, corrugated plate, laminar, flow

\section{Introduction}

Drag reduction is a very interesting topic thanks to the gain of energy that it allows. Several drag reduction techniques have been suggested by researchers in several fields (aeronautics, automotive ...).

In that sense, adding agents to fluid, especially polymers, is a good solution to reduce drag. Then, surfactants with their four types (anionic, cationic, nonionic and amphoteric) are also good drag reducers [1].

Other solutions have been proposed by adding drag reducers such as devices added at wingtips of an aircraft (flyers, flaps ...). These devices act on marginal vortices to reduce drag [2].

In addition, there are several boundary layer control methods that have been developed to reduce frictional resistance of streamlined bodies, such as the injection of a different gas [3], the acceleration of the boundary layer [4], etc. All these devices are described in detail in scientific literature.

Finally, the use of a liquid film on the surface of a body reduces its frictional resistance [5].

In this study, drag reduction is achieved by changing the shape of a flat plate in laminar flow regime. Simulations are performed by the use of the CFD software ANSYS FLUENT. This work is a preliminary study that assesses the influence of corrugations (shapes and orientations) and other patterns on drag force exerted on the plate.

\section{Numerical modeling}

This work deals with the study of a laminar incompressible fluid flow on a plate (dimensions: $1 \mathrm{~m} \times 1 \mathrm{~m}$ ) (figure 3).

The fluid (air) has a density $\rho\left(\mathrm{kg} \mathrm{m}^{-3}\right)$, a kinematic viscosity $\nu\left(\mathrm{m}^{2} \mathrm{~s}^{-1}\right)$ and a pressure $p(\mathrm{~Pa})$.

Non Dimensionalized Navier-Stokes equations in Cartesian coordinates for an incompressible fluid are:

$$
\frac{\partial \mathbf{V}}{\partial t}+(\mathbf{V} \cdot \nabla) \mathbf{V}=-\operatorname{grad} \mathbf{p}+\frac{1}{R e} \nabla^{2} \mathbf{V}
$$

In these equations, variables are non-dimensionalized by choosing the reference scales $L=1 \mathrm{~m}$ and $U=1 \mathrm{~m} / \mathrm{s}$. Where $L$ is the length of the flat plate and $U$ is the fluid freestream velocity.

The Reynolds number is defined by:

$$
R e=\frac{U L}{\nu}
$$

The drag coefficient is defined by:

$$
C_{d}=\frac{F_{t}}{\frac{1}{2} \rho U^{2} S}
$$

where $F_{t}$ is the drag force and $S$ is the surface area of the plate.

\section{Tests configuration}

The figure 1 represents the boundary conditions for the various tests. These conditions are detailed in the figure 2. 


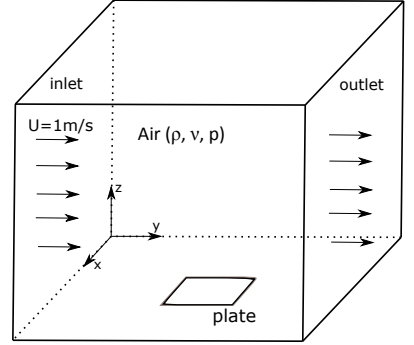

Figure 1: Boundary conditions of simulations

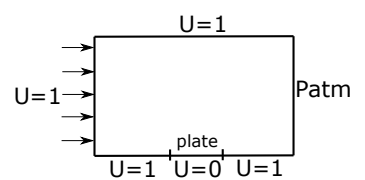

(a) Front view

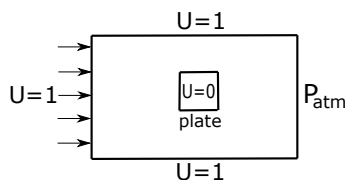

(b) Top view
Figure 2: Boundary conditions description

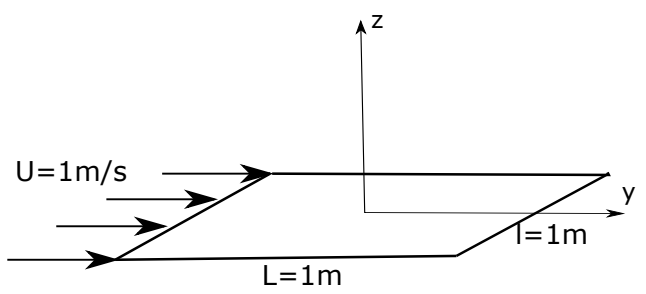

Figure 3: Flat plate exposed to airflow

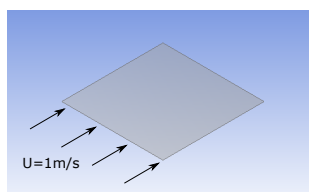

(a) Reference plate

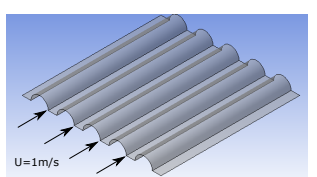

(b) Corrugated plate

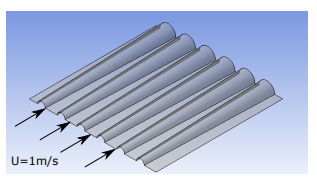

(d) Convergent corrugated plate

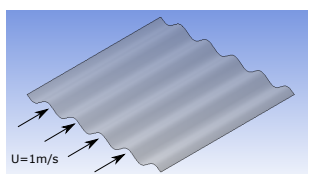

(f) Sinusoidal plate

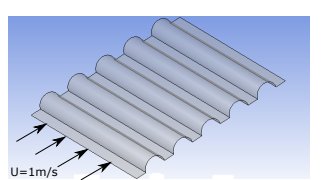

(c) Inverted corrugated plate

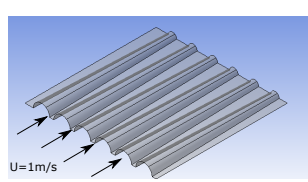

(e) Divergent corrugated plate

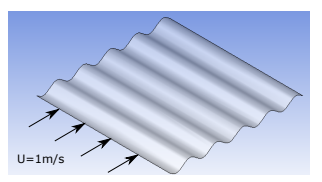

(g) Inverted sinusoidal plate
Figure 4: The studied shapes of the plate
Simulations are realized in steady state, with a calculation precision of $10^{-6}$ and their purpose is the determination of drag coefficient $C_{d}$ for each of the plate shapes.

\section{Results and discussion}

The figure 4 represents the studied shapes of the plate. They have the same dimensions $(1 \mathrm{~m} \times 1 \mathrm{~m})$ and they are subject to the same flow conditions and for which drag coefficient $C_{d}$ is calculated.

The corrugated plate (4b) and the inversed corrugated plate (4c) are identical but their flow directions are different. Similarly for the convergent corrugated plate (4d) and the divergent corrugated plate (4e). As well as for the sinusoidal plate (4f) and the inverted sinusoidal plate (4g).

Tables 1, 2 and 3 represent drag coefficients $C_{d}$ and the relative drag modifications $\frac{\Delta C_{d}}{C_{d}}$ versus the reference plate (4a) obtained for the studied shapes and for the various Reynolds numbers.

Table 1: Drag coefficient $C_{d}$ of the reference plate compared to corrugated and inverted corrugated plates

\begin{tabular}{|c|c|c|c|c|c|}
\hline \multirow{2}{*}{$\mathbf{R e}$} & \multirow{4a}{a}{} & \multicolumn{2}{|c|}{$\mathbf{4 b}$} & \multicolumn{2}{|c|}{$\mathbf{4 c}$} \\
\cline { 2 - 6 } & $\mathbf{C}_{\mathbf{d}}$ & $\mathbf{C}_{\mathbf{d}}$ & $\frac{\Delta C_{d}}{C_{d}}$ & $\mathbf{C}_{\mathbf{d}}$ & $\frac{\Delta C_{d}}{C_{d}}$ \\
\hline $\mathbf{1 0}$ & 2.305 & 3.048 & $32.2 \%$ & 4.002 & $73.6 \%$ \\
\hline $\mathbf{5 0}$ & 0.562 & 0.725 & $33.8 \%$ & 0.996 & $77.2 \%$ \\
\hline $\mathbf{1 0 0}$ & 0.325 & 0.415 & $27.7 \%$ & 0.609 & $87.4 \%$ \\
\hline $\mathbf{5 0 0}$ & 0.105 & 0.132 & $25.7 \%$ & 0.248 & $136.2 \%$ \\
\hline $\mathbf{1 0 0 0}$ & 0.068 & 0.085 & $25 \%$ & 0.182 & $167.6 \%$ \\
\hline
\end{tabular}

Table 2: Drag coefficient $C_{d}$ of the reference plate compared to convergent corrugated and divergent corrugated plates

\begin{tabular}{|c|c|c|c|c|c|}
\hline \multirow{2}{*}{$\mathbf{R e}$} & $\mathbf{4 a}$ & \multicolumn{2}{|c|}{$\mathbf{4 d}$} & \multicolumn{2}{|c|}{$\mathbf{4 e}$} \\
\cline { 2 - 6 } & $\mathbf{C}_{\mathbf{d}}$ & $\mathbf{C}_{\mathbf{d}}$ & $\frac{\Delta C_{d}}{C_{d}}$ & $\mathbf{C}_{\mathbf{d}}$ & $\frac{\Delta C_{d}}{C_{d}}$ \\
\hline $\mathbf{1 0}$ & 2.305 & 3.294 & $42.9 \%$ & 3.391 & $47.2 \%$ \\
\hline $\mathbf{5 0}$ & 0.562 & 0.780 & $38.8 \%$ & 0.776 & $38.1 \%$ \\
\hline $\mathbf{1 0 0}$ & 0.325 & 0.445 & $36.9 \%$ & 0.432 & $32.9 \%$ \\
\hline $\mathbf{5 0 0}$ & 0.105 & 0.141 & $34.3 \%$ & 0.127 & $21 \%$ \\
\hline $\mathbf{1 0 0 0}$ & 0.068 & 0.092 & $35.3 \%$ & 0.079 & $11 \%$ \\
\hline
\end{tabular}


Table 3: Drag coefficient $C_{d}$ of the reference plate compared to sinusoidal and inverted sinusoidal plates

\begin{tabular}{|c|c|c|c|c|c|}
\hline \multirow{2}{*}{$R \mathbf{R e}$} & $\mathbf{4 a}$ & \multicolumn{2}{|c|}{$\mathbf{4}$} & \multicolumn{2}{|c|}{$\mathbf{4 g}$} \\
\cline { 2 - 6 } & $\mathbf{C}_{\mathbf{d}}$ & $\mathbf{C}_{\mathbf{d}}$ & $\frac{\Delta C_{d}}{C_{d}}$ & $\mathbf{C}_{\mathbf{d}}$ & $\frac{\Delta C_{d}}{C_{d}}$ \\
\hline $\mathbf{1 0}$ & 2.305 & 2.977 & $29.2 \%$ & 2.071 & $-10.2 \%$ \\
\hline $\mathbf{5 0}$ & 0.562 & 0.703 & $25.1 \%$ & 0.527 & $-6.2 \%$ \\
\hline $\mathbf{1 0 0}$ & 0.325 & 0.399 & $22.7 \%$ & 0.314 & $-3.4 \%$ \\
\hline $\mathbf{5 0 0}$ & 0.105 & 0.123 & $17.1 \%$ & 0.107 & $1.9 \%$ \\
\hline $\mathbf{1 0 0 0}$ & 0.068 & 0.078 & $14.7 \%$ & 0.070 & $2.9 \%$ \\
\hline
\end{tabular}

As expected, drag coefficient $C_{d}$ is inversely proportional to Reynolds number $R e$ for all configurations of the plate. This is consistent with several existing formulas in the literature that express drag coefficient as a function of Reynolds of flow.

The inverted corrugated plate $(4 \mathrm{c})$ is the most unfavorable configuration. It increases hugely $C_{d}$ of the flat plate for all Reynolds numbers especially for $R e=500$ (increase of $136.2 \%$ for $R e=500$ ). That's why it's necessary to avoid such configurations in low Reynolds number designs.

Concerning the corrugated plate (4b), the convergent corrugated plate (4d) and divergent corrugated plate (4e), they always increase drag coefficient with respect to the flat plate (4a).

When Reynolds number increases, $C_{d}$ of the divergent corrugated plate (4e) decreases compared to the convergent corrugated plate (4d) and compared to the corrugated plate (4b) (for $R e=1000, C_{d}$ of the divergent corrugated plate increase by $11 \%, C_{d}$ of the convergent corrugated plate increase by $35.3 \%$ and $C_{d}$ of the corrugated plate increase by $25 \%$ compared to the flat plate). This result shows that divergent corrugations improve $C_{d}$ of simple and convergent corrugations.

The sinusoidal configuration (4f) has the lowest $C_{d}$ of all configurations ( $4 \mathrm{~b}, 4 \mathrm{c}, 4 \mathrm{~d}$ and $4 \mathrm{e}$ ), but its $C_{d}$ remains higher than the $C_{d}$ of the flat plate (4a).

Finally, the inverted sinusoidal plate $(4 \mathrm{~g})$ is the configuration that reduces drag coefficient of the flat plate. In fact, for low Reynolds numbers, the reduction of $C_{d}$ is outstanding:

- For $R e=10$, the inverted sinusoidal plate reduces remarkably drag coefficient of the flat plate $C_{d}$ from 2.305 to 2.071 (reduction of $10.2 \%$ ).

- For $R e=50$, this sinusoidal plate reduces drag coef- ficient $C_{d}$ from 0,562 to 0,527 (reduction of $6.2 \%$ ).

- For $R e=100$, it reduces it from 0,325 to 0,314 (reduction of $3.4 \%$ ).

- For the Reynolds numbers $R e=500$ and $R e=1000$, a slight increase of $C_{d}$ is observed compared to the flat plate.

This configuration will be used mainly for low Reynolds numbers since it reduces remarkably $C_{d}$ by $10.2 \%$ for $R e=10$, by $6.2 \%$ for $R e=50$ and by $3.4 \%$ for $R e=100$.

\section{Conclusion}

In this preliminary work, numerical simulations were carried out to study the effect of changing plate shape on drag reduction for a laminar incompressible air flow on this plate.

Simulations were performed by the CFD software ANSYS FLUENT.

Drag coefficients are compared between the various plate shapes. The analysis of results leads to conclude that the inverted sinusoidal plate reduces drag coefficient of the plate for low Reynolds numbers.

Future works will focus on the optimisation of flat plate shape to reduce drag coefficient for high Reynolds number flows and the extension to turbulent flows.

\section{References}

[1] F. Hadri, Contribution à l'étude de la réduction de la traînée en écoulement turbulent d'une solution de CTAC, Ph.D. Dissertation, University of Caen, 2008.

[2] R.V.J. Viard, Dispositifs fluidiques de Contrôle actif d'écoulements à base de Microsystèmes MagnétoElectro-Mécaniques (MMEMS) (conception, réalisation, tests), Ph.D. Dissertation, Ecole Centrale in Lille, 2010.

[3] S.A. Mäkiharju, S.L. Ceccio, On multi-point gas injection to form an air layer for frictional drag reduction, Ocean Eng. 147 (2018) $206-214$.

[4] K. Gersten, H. Schlichting, Boundary-LayerTheory Ninth Edition, Springer-Verlag, Berlin Heidelberg, Germany, 2017.

[5] N. Klyuev, Y. Kryukov, A. Gimadiev, Reducing friction drag on flat plates, Procedia Engineering 176 (2017) $661-668$. 